\title{
A MODEL OF DENSE FLUIDS
}

\author{
R. F. STREATER \\ Department of Mathematics, King's College, Strand, London \\ E-mail: ray.streater@kcl.ac.uk \\ and \\ Department of Physics, University of Madeira, Funchal, Portugal
}

\begin{abstract}
We obtain coupled reaction-diffusion equations for the density and temperature of a dense fluid, starting from a discrete model in which at most one particle can be present at each site. The model is constructed by the methods of statistical dynamics. We verify that the theory obeys the first and second laws of thermodynamics. Some remarks on measurement theory for the position of a particle are offered.
\end{abstract}

1. Introduction. In recent work $[9,11]$ I describe a general method for constructing nonlinear dynamical systems obeying the first and second laws of thermodynamics. In the last Chapter of [11], some connections with information theory are mentioned. The outlook of the theory has been further influenced by information geometry, both classical $[1,13]$ and quantum $[7,8,4,6,5,12]$. The systems are set up within a probability theory, either classical or quantum; the dynamics is then described by an orbit through the set of states; to formulate the first law of thermodynamics we need to specify the energy, which is a random variable in the classical case and a self-adjoint operator in the quantum case. In either case the first law is expressed by saying that the mean energy is constant on the orbit. The second law is expressed by saying that the entropy, of Shannon or von Neumann, is a nondecreasing function along the orbit. In $[9,11]$ the simple case of discrete space and time is done in detail; the orbit is then given by an iterated nonlinear map on the space of components of the probabilities. In [14] the programme is carried out for a particle in a potential, moving in a dense liquid. The formal limit to a spacetime continuum is taken, for both a classical and a quantum model. We arrive at coupled nonlinear heat-diffusion equations for the temperature and density, which complement the Smoluchowski equation by a further equation if the temperature is not constant. In [15] we show that the limiting equations obey the first and second laws of thermodynamics, expressed in terms of the formal continuum limit of the mean energy and the entropy. In

1991 Mathematics Subject Classification: Primary 80; Secondary 94.

The paper is in final form and no version of it will be published elsewhere. 
[16] we show that for small times and under certain conditions on the initial state and the potential, there exists a solution to the coupled equations for which the density is nonnegative and the temperature field is positive.

In these models, any number of particles can occupy a given region, and their density obeys the heat equation with drift; the fluctuations therefore obey a stochastic differential equation driven by Brownian motion, and it is reasonable to call them Brownian particles. We find that the friction is caused by Bremsstrahlung, and that the heat lies in the ambient liquid, rather than in the thermal motion of the particles. This shows up in that the heat capacity $C$ is not proportional to the particle density, and $C$ is interpreted as the heat-capacity of the ambient fluid. In the construction, the correlation between a particle and the temperature is treated as a fast variable, and is put to zero in the coarse graining. It is thus not a good model for a single Brownian particle, which keeps the correlation between its position and the heat it has produced. We therefore titled the model "A Gas of Brownian particles..."; this postulates that the correlation between one particle and the heat produced by all the particles vanishes.

In the present paper we study a classical version of a model of a fluid of particles in a potential where there is a maximum possible density. We start, in Sect 2., with the discrete model, taken from [10]; the occupation number of each site is taken to be 0 or 1 , so it represents a particle with a hard core; the particle carries kinetic energy that is quantised into units; it moves through a potential $V$ which can depend on position but not time. We complete the analysis of [10] and [11] page 69 , by finding the equation for the temperature, and taking the continuum limit of the coupled system. The limit is taken in such a way that the maximum permitted density $\rho$ is $\rho_{\max }$. Let $\Theta(x, t)$ be the temperature and $F(x)=-\nabla V$ the force on a particle when it is at $x$. We get the equations

$$
\begin{aligned}
\frac{\partial \rho}{\partial t} & =\lambda\left\{\nabla^{2} \rho+\operatorname{div}\left(\nabla V \rho\left(1-\rho / \rho_{\max }\right) / \Theta(x, t)\right)\right\} \\
& =-\operatorname{div} j, \quad \text { say; } \\
\frac{\partial(\rho \Theta)}{\partial t} & =\lambda \operatorname{div}\left[\rho\left(1-\rho / \rho_{\max }\right) \nabla \Theta\right]-\nabla V . j-\operatorname{div}(\Theta j) .
\end{aligned}
$$

We see that the energy-density is proportional to the particle density; the system can therefore describe a dense fluid; there is a flow of heat due to diffusion due to temperature gradients, coming from the first term in the right hand side of (3), and a further heat drift coming from the last term $-\operatorname{div}(\Theta j)$, which is the loss due to the convection by the particle-current.

The model obeys the Einstein relation (between the diffusion constant, the force, the terminal velocity and the temperature), as well as both laws of thermodynamics. The Gibbs state is a stationary solution if the system is put in a box with no heat-flow and no particle current normal to the boundary. The heat capacity is proportional to the particle density. This is a good property for a dense fluid (I thank Prof. Benilov for useful comments on this point); but there are some oversimplifications in the model: the density of states is not realistic for a system in three dimensions, and the scattering rate, $\lambda$, is taken to be independent of the kinetic energy of the particle. This leads to the diffusion 
constant's being independent of the temperature.

That the continuum equations obey both laws of thermodynamics seems to be quite a miracle, but it should come as no surprise; the discrete models are constructed so as to do just this, and the continuum versions of the entropy and the energy are just the continuum limits of the discrete versions. We must drop some divergent quantities in the entropy limit, but these are time independent divergences, and do not spoil the second law.

In classical statistical dynamics, the system is described by a sample space $\Omega$, taken to be countable. The set of probability measures on $\Omega$ is the state-space $\Sigma(\Omega)$. The energy $\mathcal{E}$ is a random variable, with values $E$. The energy divides $\Omega$ into a disjoint union of "energy-shells", thus: $\Omega=\cup_{E} \Omega_{E}$, where

$$
\Omega_{E}=\{\omega \in \Omega: \mathcal{E}(\omega)=E\} .
$$

In models with local structure, such as those in this paper, the sample space is a product of sample spaces, one for each $x \in \Lambda$; thus:

$$
\Omega=\prod_{x \in \Lambda} \Omega_{x}
$$

We interpret $\Lambda$ as the space in which the system sits, and is usually a finite subset of the lattice $\mathbf{Z}^{\nu}$, where $\nu$ is the number of space-dimensions in the model. Time is also discrete, and one time-step in the dynamics is described, in the first place, by a bistochastic map $T^{d}: \Sigma(\Omega) \rightarrow \Sigma(\Omega)$. It is written as the dual to a normal bistochastic map $T$ on the observable algebra $L^{\infty}(\Omega)$ of bounded functions on the sample space. To ensure that mean energy is conserved, we require that $T$ maps the indicator functions $\chi_{E}$ on each $\Omega_{E}$ to itself, thus: $T \chi_{E}=\chi_{E}$ for all energies $E$. With this, one time step then conserves the mean energy. Since $T$ is bistochastic, its dual $T^{d}$ increases the entropy (or leaves it unchanged). We thus have all the ingredients of the two laws of thermodynamics. However, the ergodic properties of such a system are not strong enough to lead us to thermal equilibrium. At best, if we start with a state with a given energy $E$, then iteration of the above one step can lead to the microcanonical state on the energy-shell $\Omega_{E}$; but it cannot lead to a canonical state. We need more randomness to get this, and we use an idea from information geometry [2], developed in [13]; let us specify a list $\mathcal{X}=\left\{X_{1}, \ldots, X_{n}\right\}$ as slow variables. The means $\mu_{i}, i=1, \ldots, n$ of these variables will be the objects in terms of which the dynamics will be written. Among the slow variables will be the constants, and the energy. In this paper we include the local potential energy and the local kinetic energy among the slow variables. In classical probability, the $X_{i}$ are random variables; in quantum probability, they are (not necessarily commuting) self-adjoint operators. Given any state $p$ of the system, we denote by $p . X$ the mean of $X$ in the state $p$. We seek a state, $Q p$, which has the same means for the slow variables as has $p$, and has the maximum entropy among all states with this property. Thus

$$
\begin{gathered}
\qquad p \cdot X_{i}=p \cdot X_{i}, \quad i=1, \ldots, n ; \\
S(Q p) \geq S(q) \text { for all } q \in \Sigma \text { such that } q \cdot X_{i}=p \cdot X_{i}, i=0,1,2 \ldots
\end{gathered}
$$

In discrete classical systems [1], and some quantum systems $[12,4,7], Q p$ is unique and 
is given as a point in an exponential family, which make up a statistical manifold $S_{n-1}$; to any point $p \in \Sigma$, in geometrical terms the point $Q p$ is the orthogonal projection of $p$ onto $S_{n-1}$ along a $\stackrel{-1}{\nabla}$-geodesic. In statistical terms, $Q p$ is the best estimate for $p$ among all states on the manifold. In neural nets, we can describe $p$ as the input and $Q p$ as the inference made or memory recalled by the brain. In thermodynamics it is reasonable to regard $Q p$ as the state obtained by thermalising all except the slow variables; indeed, it is known that $Q p$ is the state in local thermodynamic equilibrium which has the same slow means as $p$. The map $Q$ is not obtainable from a Hamiltonian; it is nonlinear, and represents the dissipation not accounted for by the linear stochastic map $T$. It is, mathematically, a generalisation of the Stosszahlansatz.

One time step of statistical dynamics, expressing the change in the state in a time $d t$, is given by $p \mapsto Q T^{d} p$; by construction, this conserves the mean energy and increases the entropy except at a fixed point; this is the basis for our confidence that models constructed in this way will obey both laws of thermodynamics.

\section{The discrete model}

2.1. Classical particle with potential and kinetic energy. This is a development of the model described in [10] and [11], p. 69. Consider space to be one-dimensional for simplicity, and discrete, so that $\Lambda=\mathbf{Z}$. The distance between points will be taken to be $\ell$, of molecular size. Each site can either be occupied or not, so we are describing a hard-core model with maximal density $f_{\max }=\ell^{-1}$. If a particle is present at $x \in \Lambda$, its kinetic energy is assumed to be a nonnegative integer multiple of a basic quantum $\epsilon$. Thus the kinetic energy is $k \epsilon, k=0,1,2 \ldots$. In this simple model, we do not take account of any multiplicity in the states of a given kinetic energy. That is, we put the density of states equal to 1 . We are using the field description, rather than the particle description. This ensures that the particles are indistinguishable, and avoids the Gibbs paradox. The above remarks are summarised by saying that the sample space of the theory is the product set

$$
\Omega=\prod_{x \in \Lambda} \Omega_{x}
$$

where

$$
\Omega_{x}=\{\emptyset, 0,1, \ldots\} .
$$

Thus, a sample point is a function $\omega: \Lambda \rightarrow \cup_{x} \Omega_{x}$ such that $\omega_{x} \in \Omega_{x}$; in simple terms, a sample point is the family $\left\{\omega_{x}\right\}_{x \in \Lambda}$. If $\omega_{x}=\emptyset$, then there is no particle at $x$; if $\omega_{x}=k_{x}$, then there is a particle at $x$ with kinetic energy $k_{x} \epsilon$. The kinetic energy is thus the random variable

$$
\mathcal{K}(\omega)=\sum_{x: \omega_{x} \neq \emptyset} \epsilon k_{x} .
$$

The potential energy of a particle at $x$ is taken to be a given time-independent function $V(x)$, an integer multiple $v_{x}$ of $\epsilon$. The potential energy of the model is thus the random variable

$$
\mathcal{V}(\omega)=\sum_{x: \omega_{x} \neq \emptyset} V(x)=\sum_{x: \omega_{x} \neq \emptyset} \epsilon v_{x}
$$


This can be written

$$
\mathcal{V}=\sum_{x} \epsilon v_{x} n_{x}
$$

where $n_{x}(\omega)$ is the number of particles at $x$ when the configuration is $\omega$; thus $n_{x}=0$ if $\omega_{x}=\emptyset$, and $n_{x}=1$ if $\omega_{x} \neq \emptyset$. The total energy is the random variable

$$
\mathcal{E}=\mathcal{K}+\mathcal{V} \text {. }
$$

It is this, rather than $\mathcal{K}$ and $\mathcal{V}$ separately, that is to be conserved (in mean) by the dynamics. Thus, in the linear part of the dynamics, a transition can occur only between two points in the same energy-shell. We shall limit our model to allow only nearestneighbour transitions in one time-step. The typical transition is then from a state with one particle at $x$ with kinetic energy $k \epsilon$ and a hole at $x-\ell$, to the state with a particle at $x-\ell$ with kinetic energy $\epsilon\left(k+v_{x}-v_{x-\ell}\right)$, and a hole at $x$ (the rest of the configuration being the same as in $\omega)$. The inverse transition can also occur, and is taken to have the same probability; this makes the stochastic matrix bistochastic, and ensures that entropy is a monotonic increasing function of time.

The state (at time $t$, say) is given by a probability distribution $p(\omega)$, and the state at a point $x \in \Lambda$ is then determined by the marginal distribution on $\Omega_{x}$ :

$$
p_{x}\left(\omega_{x}\right)=\sum_{\omega^{\prime}: \omega_{x}^{\prime}=\omega_{x}} p\left(\omega^{\prime}\right) .
$$

A state of the form of a product state of its marginals,

$$
p(\omega)=\prod_{x} p_{x}\left(\omega_{x}\right)
$$

is said to be independent over $\Lambda$. If $p$ is independent over $\Lambda$ and, further, each marginal $p_{x}$ of $p$ is a grand canonical state then we say that $p$ is in local thermodynamic equilibrium $(L T E)$. Since $n_{x}$ can only take the two values 0,1 , any grand canonical state can be written in terms of $q_{x}$ and $p_{x}(k)$, thus:

$$
\begin{aligned}
& p\left(\omega_{x}=\emptyset\right)=q_{x}=1-p_{x}, \\
& p\left(\omega_{x}=k\right)=p_{x}(k)=p_{x}\left(1-e^{-\beta_{x} \epsilon}\right) e^{-\beta_{x} \epsilon k} .
\end{aligned}
$$

We see that

$$
p_{x}=\sum_{k \geq 0} p_{x}(k) .
$$

In the model under discussion, we take as the slow variables the number of particles at each $x$, namely, all the $n_{x}$, together with the sure random variables and the kinetic energy at each site. These span the real vector space $\mathcal{X}=\operatorname{Span}\left\{1, \mathcal{K}_{x}, n_{x}: x \in \Lambda\right\}$. The thermalising map $Q$ therefore conserves the means of each $n_{x}$, namely the marginal probability $p_{x}$, and the mean kinetic energy, $K(x)$, related to to beta in LTE by

$$
K(x)=p \cdot \mathcal{K}_{x}=\epsilon p_{x} \sum_{k}\left(1-e^{-\beta_{x} \epsilon k}\right) k e^{-\beta_{x} \epsilon k}=\epsilon p_{x}\left(e^{\beta_{x} \epsilon}-1\right)^{-1} .
$$

It is known that the map $Q$, which maximises the entropy subject to conserving all the local densities and energies, in this case takes a state $p$ to a state in LTE; namely, the $L T E$-state with the same mean density $p . n_{x}$ and kinetic energy $p . \mathcal{K}_{x}$. In [11] we considered 
the case when the external field was constant in space, and formulated a transition matrix in one dimension from the site $x$ to $x+\ell$ and vice versa. The same discussion leads to a similar form for the transition, in one time-step, from the site $x$ to and from the site $x-\ell$. In the present model, the difference in potential energy is not constant, but is $\epsilon w_{x}$, where

$$
w_{x}=v_{x+\ell}-v_{x}
$$

Recall that we assume that each $v_{x}$ is an integer; we shall take the case when $w_{x} \geq 0$; the other case is argued similarly. Similarly to [11] we can show that a possible transition in one time-step, within an energy-shell, is given by the operator $T$, where

$$
\begin{aligned}
(T p)_{x}(k) & =p_{x}(k)-\lambda\left(k-w_{x}\right) p_{x}(k) q_{x+\ell}+\lambda\left(k-w_{x}\right) p_{x+\ell}\left(k-w_{x}\right) q_{x} \\
& -\lambda(k) p_{x}(k) q_{x-\ell}+\lambda(k) p_{x-\ell}\left(k+w_{x-\ell}\right) q(x) .
\end{aligned}
$$

Here $\lambda$ is the transition probability; we shall choose it to be independent of the occupation number, as far as possible. However it must be zero for negative argument; this corresponds to the constraint that a particle cannot move one step up against the external field (and conserve energy) if it does not possess at least as much kinetic energy as the potential difference. The terms in the first line of (19) correspond to the probability $p_{x}(k)$ that site $x$ is occupied with a particle of kinetic energy $\epsilon k$, times the probability $q_{x+\ell}$ that the site $x+\ell$ is empty, which, times the rate $\lambda$ gives the loss to the state; similarly, the gain to the state with the same rate is the probability that $x+\ell$ is occupied by a particle of kinetic energy $\epsilon\left(k-x_{x}\right)$, times the probability that the site $x$ is unoccupied. Similarly, the terms in the second line of (19) have a natural interpretation. We show in [11] that the operator $T$ is a bistochastic matrix, for small positive $\lambda$.

2.2. Discrete equation for the density. Let us now calculate the densities at time $t+d t$. Using eq. (16) we see that the change due to the linear part of the dynamics is

$$
\begin{aligned}
p(x, t+d t) & =\sum_{k} p_{x}(k, t+d t)=\sum_{k}(T p)_{x}(k) \\
& =\sum_{k}\left\{p_{x}(k)-\lambda\left(k-w_{x}\right) p_{x}(k)(1-p(x+\ell))\right. \\
& +\lambda\left(k-w_{x}\right) p_{x+\ell}\left(k-w_{x}\right)(1-p(x))-\lambda(k) p_{x}(k)(1-p(x-\ell)) \\
& \left.+\lambda(k) p_{x-\ell}\left(k+w_{x-\ell}\right)(1-p(x))\right\}
\end{aligned}
$$

Here, $p_{x}(k)$ is the grand canonical state eq.(17), and $\lambda(r)=\lambda$ for $r \geq 0$, and zero for negative $r$. The sums in eq. (20) are:

$$
\begin{aligned}
\sum_{k} p_{x}(k) & =p(x) ; \\
\sum_{k} \lambda\left(k-w_{x}\right) p_{x}(k)(1-p(x+\ell)) & =-\lambda(1-p(x+\ell)) \sum_{k \geq w_{x}} p_{x}(k) \\
& =-\lambda(1-p(x+\ell)) \exp \left\{-\beta_{x} w_{x} \epsilon\right\} \sum_{k} p_{x}(k) \\
& =-\lambda p(x)(1-p(x+\ell)) \exp \left\{-\beta_{x} w_{x} \epsilon\right\} .
\end{aligned}
$$


Similarly,

$$
\begin{aligned}
\sum_{k} \lambda\left(k-w_{x}\right) p_{x+\ell}\left(k-w_{x}\right)(1-p(x)) & =\lambda p(x+\ell)(1-p(x)) \\
-\sum_{k} \lambda(k) p_{x}(k)(1-p(x-\ell)) & =-\lambda p(x)(1-p(x-\ell)) \\
\sum_{k} \lambda(k) p_{x-\ell}\left(k+w_{x-\ell}\right)(1-p(x)) & =\lambda \exp \left\{-\beta_{x-\ell} w_{x-\ell} \epsilon\right\} p(x-\ell)(1-p(x)) .
\end{aligned}
$$

Now the map $Q$ preserves the mean of $n(x)$, namely $p(x)$, for all $x$. Thus, collecting the above sums, the combined map $Q T$ leads to one time-step for $p$ :

$$
\begin{aligned}
p(x, t+d t) & =p(x)-\lambda p(x)(1-p(x+\ell)) \exp \left\{-\beta_{x} w_{x} \epsilon\right\} \\
& +\lambda p(x+\ell)(1-p(x))-\lambda p(x)(1-p(x-\ell)) \\
& +\lambda p(x-\ell)(1-p(x)) \exp \left\{-\beta_{x-\ell} w_{x-\ell} \epsilon\right\} .
\end{aligned}
$$

2.3. Discrete equation for the kinetic energy. The other slow variables are the kinetic energy density at each point, $\mathcal{K}(x)$. The means $K(x, t+d t)$ of $\mathcal{K}(x)$ in the state $T p$ are preserved by the map $Q$. Now, $K(x, t)=p \cdot \mathcal{K}(x)=\epsilon \sum k p_{x}(k)$, so, looking at eq. (20) we get

$$
\begin{aligned}
K(x+d t) & =Q T p \cdot \mathcal{K}(x)=T p \cdot \mathcal{K}(x)=\epsilon \sum_{k=0} k T p_{x}(k) \\
& =\epsilon \sum_{k=0}\left\{k p_{x}(k)-k \lambda\left(k-w_{x}\right) p_{x}(k) q_{x+\ell}\right. \\
& +k \lambda\left(k-w_{x}\right) p_{x+\ell}\left(k-w_{x}\right) q(x) \\
& \left.-k \lambda(k) p_{x}(k) q_{x-\ell}+k \lambda(k) p_{x-\ell}\left(k+w_{x-\ell}\right) q(x)\right\}
\end{aligned}
$$

In the term $-k \lambda\left(k-w_{x}\right) p_{x} q_{x+\ell}, \lambda$ is zero when the argument is negative; the sum therefore begins at $w_{x}$ instead of 0 . Change the summation label from $k$ to $k+w_{x}$. We now use that $p$ is in $L T E$, and so

$$
p_{x}\left(k+w_{x}\right)=e^{-\beta_{x} w_{x} \epsilon} p_{x}(k) .
$$

Similarly, in the last term we may use the relation

$$
p_{x-\ell}\left(k+w_{x-\ell}\right)=e^{-\beta_{x-\ell} w_{x-\ell} \epsilon} p_{x-\ell}(k) .
$$

Then we may do all the sums over $k$, to get

$$
\begin{aligned}
K(x, t+d t) & =K(x, t)+\lambda\left\{-e^{-\beta_{x} w_{x} \epsilon} K(x, t)-w_{x} e^{-\beta_{x} w_{x} \epsilon} p(x) \epsilon\right. \\
& +p(x+\ell) e^{-\beta_{x} w_{x} \epsilon} K(x, t)+p(x+\ell) w_{x} e^{-\beta_{x} w_{x} \epsilon} p(x) \epsilon \\
& +K(x+\ell, t)+w_{x} p(x+\ell) \epsilon-p(x) K(x+\ell, t) \\
& -w_{x} p(x) p(x+\ell) \epsilon-K(x, t)+p(x-\ell) K(x, t) \\
& \left.+e^{-\beta_{x-\ell} w_{x-\ell} \epsilon} K(x-\ell, t)-p(x) e^{-\beta_{x-\ell} w_{x-\ell} \epsilon} K(x-\ell, t)\right\} .
\end{aligned}
$$

One can verify that the mean total energy $E=\sum_{x}(K(x)+\epsilon v(x) p(x))$ is conserved in time, as it should by construction. It is harder to prove directly that entropy increases; this will be achieved for the continuum limit in Sect. 4. 


\section{The continuum limit}

3.1. Continuum equation for the density. We start with the discrete-time-discretespace evolution equations for the slow variables. These are (cf. eq. (20))

$$
\begin{aligned}
p(x, t+d t) & =p(x)+\lambda\left\{p(x+\ell)(1-p(x))-p(x)(1-p(x+\ell)) e^{-\beta_{x} w_{x} \epsilon}\right. \\
& -p(x)+p(x) p(x-\ell)\left(1-e^{-\beta_{x-\ell} w_{x-\ell} \epsilon}\right) \\
& \left.\left.+p(x-\ell) e^{-\beta_{x-\ell} w_{x-\ell} \epsilon}\right)\right\}
\end{aligned}
$$

and (cf. eq. (30)):

$$
\begin{aligned}
K(x, t+d t) & =K(x)+\lambda\left\{-e^{-\beta_{x} w_{x} \epsilon} K(x)-w_{x} p(x) \epsilon e^{-\beta_{x} w_{x} \epsilon}\right. \\
& +p(x+\ell) K(x) e^{-\beta_{x} w_{x} \epsilon}+p(x+\ell) w_{x} p(x) \epsilon e^{-\beta_{x} w_{x} \epsilon} \\
& +w_{x} p(x+\ell) \epsilon-p(x) K(x+\ell)-w_{x} p(x) p(x+\ell) \epsilon \\
& -K(x)+p(x-\ell) K(x)+K(x-\ell) e^{-\beta_{x-\ell} w_{x-\ell} \epsilon} \\
& \left.-p(x) K(x-\ell) e^{-\beta_{x-\ell} w_{x-\ell} \epsilon}+K(x+\ell)\right\} .
\end{aligned}
$$

Recall that $p(x), K(x)$ denote the values at time $t$. We want to take a limit of these, as $d t \rightarrow 0, \ell \rightarrow 0$ in such a way that we get the Fick equation [3] for the transport of particles, together with further terms. We do this by assuming that there is a set of smooth functions of $x, t$, say $p(x, t), K(x, t)$ and $V(x)$, which, when evaluated at the discrete space-time points $\ell \mathbf{Z} \times d t \mathbf{Z}$, obey these equations, with an error of order $\ell^{3}$. We then find the partial differential equations that this implies. It is clear that $\epsilon$ is $O(\ell)$ in the limit $\ell \rightarrow 0$, as it is the smallest amount of potential energy that can be converted into heat as we move a distance $\ell$, and this is bounded by $|\nabla V|_{\infty} \ell$. So we write, to order $\ell^{2}, \epsilon \ell$ or $\epsilon^{2}$ :

$$
\begin{aligned}
p(x+\ell) & =p+\ell p^{\prime}+\ell^{2} / 2 p^{\prime \prime} ; \\
p(x-\ell) & =p-\ell p^{\prime}+\ell^{2} / 2 p^{\prime \prime} ; \\
e^{-\beta_{x} w_{x} \epsilon} & =1-\beta w \epsilon+\beta^{2} w^{2} \epsilon^{2} / 2 ; \\
e^{-\beta_{x-\ell} w_{x-\ell} \epsilon} & =1-\left(\beta-\ell \beta^{\prime}\right)\left(w-\ell w^{\prime}\right) \epsilon+\epsilon^{2} \beta^{2} w^{2} / 2 .
\end{aligned}
$$

Here and later, $p, \beta$ etc. denote $p(x), \beta_{x}$ etc. Substitution into eq. (31) gives

$$
\begin{aligned}
p(x, t+d t) & =p+\lambda\left\{\left(p+\ell p^{\prime}+\frac{\ell^{2} p^{\prime \prime}}{2}\right)(1-p)\right. \\
& -p\left(1-p-\ell p^{\prime}-\ell^{2} p^{\prime \prime} / 2\right)\left(1-\beta w \epsilon+\beta^{2} w^{2} \epsilon / 2\right)-p \\
& \left.+p\left(p-\ell p^{\prime}\right)\left(\beta-\ell \beta^{\prime}\right)\left(w-\ell w^{\prime}\right) \epsilon\right)-\frac{\beta^{2} w^{2} \epsilon^{2}}{2} \\
& \left.+\left(p-\ell+\ell^{2} p^{\prime \prime} / 2\right)\left(1-\left(\beta-\ell \beta^{\prime}\right)\left(w-\ell w^{\prime}\right) \epsilon+\frac{\beta^{2} w^{2} \epsilon^{2}}{2}\right)\right\} .
\end{aligned}
$$

Write out the coefficients of $1, \ell, \epsilon, \ell^{2}, \epsilon \ell$ and $\epsilon^{2}$ in turn. We find, respectively, $0,0,0, p^{\prime \prime}$, $\nabla\left\{\left(p-p^{2}\right) \beta w\right\}, 0$. So to this approximation, the dynamical equation for $p$ is

$$
p(x+d t)-p(x)=\lambda\left\{\ell^{2} p^{\prime \prime}+\nabla\left\{\left(p-p^{2}\right) \beta w\right\} \ell \epsilon\right\} .
$$

Now, in the diffusion limit, we let $\ell$ and $d t$ go to zero, such that $\ell^{2} d t$ is constant. As $\lambda$ is 
anyway a free parameter, we might as well put $\ell^{2} d t=1$. Also, $w \epsilon / \ell=\nabla V$; thus we get the equation

$$
\frac{\partial p}{\partial t}=\lambda\left\{p^{\prime \prime}+\frac{\partial\left[p(1-p) \beta V^{\prime}\right]}{\partial x}\right\} .
$$

The macroscopic quantity is really the density $\rho=m p / \ell$, where $m$ is the mass of an individual particle. So dividing the equation by $\ell$ before taking the limit gives:

$$
\frac{\partial \rho}{\partial t}=\lambda\left\{\rho^{\prime \prime}+\frac{\partial}{\partial x}\left[V^{\prime} \beta \rho(1-p)\right]\right\} .
$$

Now $p=(p m / \ell) /(m / \ell)=\rho / \rho_{\max }$, where $\rho_{\max }$ is the maximum possible density. In more than one dimension we proceed similarly, to get

$$
\frac{\partial \rho}{\partial t}=\lambda\left\{\nabla^{2} \rho+\operatorname{div}\left[\beta \rho\left(1-\rho_{\max }\right) \nabla V\right]\right.
$$

The right-hand side is a pure divergence; putting

$$
j(x, t)=-\lambda\left\{\nabla \rho+\beta \rho\left(1-\rho / \rho_{\max }\right) \nabla V\right\}
$$

we get the conservation law

$$
\frac{\partial \rho}{\partial t}+\operatorname{div} j=0 \text {. }
$$

3.2. Continuum equation for the temperature. For the kinetic energy, we start with eq. (30), and similarly expand everything up to second order, that is, up to $\ell^{2}, \ell \epsilon$ and $\epsilon^{2}$. There are four terms of zeroth order involving the Boltzmann factor, which can be paired as

$$
-e^{-\beta_{x} w_{x} \epsilon} K(x)+e^{-\beta_{x-\ell} w_{x-\ell \epsilon}} K(x-\ell)
$$

and the similar pair with an extra factor of $p$ :

$$
p(x+\ell) e^{-\beta_{x} w_{x} \epsilon} K(x)-p(x) e^{-\beta_{x-\ell} w_{x-\ell} \epsilon} K(x) .
$$

The term in eq. (43) of order $\epsilon^{2}$ is obtained by expanding the exponentials up to second order; we get

$$
\begin{aligned}
& \left\{-1 / 2 \beta_{x}^{2} w_{x}^{2} K(x)+\beta_{x-\ell}^{2} w_{x-\ell}^{2} K(x-\ell)\right\} \epsilon^{2} \\
& =-\left(\epsilon^{2} / 2\right) \ell\{(\beta w K)(x)-(\beta w K)(x-\ell)\} / \ell=O\left(\epsilon^{2} \ell\right)
\end{aligned}
$$

as $\beta w K$ is differentiable. So these terms of second order cancel. Similarly, the second order terms in eq. (44) cancel. It is therefore enough to expand the Boltzmann factors to first order. Then eq. (30) becomes, to this order,

$$
\begin{aligned}
K(x, t+d t) & =K+\lambda\{-(1-\beta w \epsilon) K-(1-\beta w \epsilon) w p \epsilon \\
& +p(x+\ell) K(1-\beta w \epsilon)+p(x+\ell) w \epsilon(1-\beta w \epsilon) p+K(x+\ell) \\
& +w p(x+\ell) \epsilon-p K(x+\ell)-w p p(x+\ell) \epsilon-K+p(x-\ell) K \\
& \left.+\left(1-\beta_{x-\ell} w_{x-\ell} \epsilon\right) K(x-\ell)-p\left(1-\beta_{x-\ell} w_{x-\ell} \epsilon\right) K(x-\ell) .\right\} \\
& =K+\lambda\{K(x+\ell-2 K(x)+K(x-\ell) \\
& +w \epsilon(p(x+\ell)-p)+\epsilon\left(\beta w K-\beta_{x-\ell} w_{x-\ell} K(x-\ell)\right) \\
& +\beta w^{2} \epsilon^{2} p(1-p(x+\ell))-\epsilon\left(p(x+\ell) \beta w K-p \beta_{x-\ell} w_{x-\ell} K(x-\ell)\right) \\
& +p(x+\ell) K-p K(x+\ell)+p(x-\ell) K-p K(x-\ell)\} .
\end{aligned}
$$


Now to order $\ell^{2}$

$$
p(x+\ell) K-p K(x+\ell)+p(x-\ell) K-p K(x-\ell)=\ell^{2}\left(p^{\prime \prime} K-p K^{\prime \prime}\right)
$$

and so the equation becomes

$$
\begin{aligned}
K(x, t+d t) & =K+\lambda \ell^{2}\left\{\frac{\partial^{2}(K)}{\partial x^{2}}+\frac{w \epsilon}{\ell} \frac{\partial p}{\partial x}+\frac{\epsilon}{\ell} \frac{\partial(K \beta w)}{\partial x}\right. \\
& \left.-\frac{\epsilon}{\ell} \frac{\partial(p K \beta w)}{\partial x}+\beta\left(\frac{w \epsilon}{\ell}\right)^{2} p(1-p)+p^{\prime \prime} K-p K^{\prime \prime}\right\}
\end{aligned}
$$

Now $w \epsilon / \ell=\nabla V$, and $K \beta=p$ up to errors $O(\ell)$; we may thus replace $K$ with $\Theta p$; we also put $\ell^{2} / d t=1$. Then rearranging terms, we get

$$
\frac{\partial(p \Theta)}{\partial t}=\lambda\left\{\frac{\partial(\Theta \nabla p)}{\partial x}+\frac{\partial}{\partial x}(\nabla \Theta p(1-p))-\nabla V \cdot j+\nabla(\nabla V p(1-p))\right\} .
$$

Here, the mass current $j$ is given by eq. (41). In several dimensions, and putting in $\rho=m p / \ell$, and $\rho_{m}=\rho_{\max }$, we get

$$
\frac{\partial(\rho \Theta)}{\partial t}=\lambda \operatorname{div}\left[\rho\left(1-\rho / \rho_{m}\right) \nabla \Theta\right]-\operatorname{div}(\Theta j)-j . \nabla V .
$$

It is interesting that a term, of second order in the space derivatives, occurs automatically in the equation for the heat, unlike in the simpler models. This represents the diffusion of heat, and is zero if $p=0$ or $p=1$, showing that there is no diffusion of heat if the density is zero. Let $J=j / m$ be the number current; then $\Theta J$ represents the convection of heat due to the particle current. The term $-J . \nabla V=J . F$ is the heat-source.

\section{The laws of thermodynamics}

4.1. The first law. The discrete system of equations (31) and (30) were constructed so that the mean energy is conserved in time. Indeed, the linear part of the dynamics causes transitions only between states of the same energy, and the nonlinear part, the map $Q$, conserves the mean energy, since $\mathcal{K}+\mathcal{V}$ is in the space of slow variables. This indicates, but does not prove, that the continuum limit of the mean energy is conserved by the continuum dynamics. The conservation might fail if some terms (say of order $\ell^{3}$ ), that diverge when integrated over space, are dropped in the limit. However, it is easy to verify that the continuum limit of the total energy, namely

$$
E=\int f(x, t) V(x) d x+\int f(x, t) \Theta(x, t) d x
$$

is conserved in time, where $f=p / \ell$. Indeed, the equation of motion for the heat energy is a pure divergence on the r.h.s., except for the term $-\nabla V . j$, which is the source of the equation; this term is exactly equal to the loss of power by the potential energy, as the particle drifts under the force $-\nabla V$. In detail, put $f_{m}=\rho_{\max } / m=1 / \ell$, and $J=j / m$; then the rate of change of the total energy is

$$
\begin{aligned}
\frac{d E}{d t} & =\int f V d x+\int(f \Theta) d x \\
& =-\int \operatorname{div} J V d x+\int\left[\operatorname{div}\left\{\lambda f\left(1-f / f_{m}\right) \nabla \Theta-\Theta J\right\}-\nabla V . J\right] d x .
\end{aligned}
$$


We impose the conditions that J.n and $\nabla \Theta$ vanish on the boundary, where $n$ is the outward normal; otherwise, there is a flow of energy through the boundary, and the first law will need modification. So we may integrate by parts, and ignore the surface terms. Since then $\int \operatorname{div}(J \Theta) d x=0$, and $\int \operatorname{div}\left(f\left(1-f / f_{m}\right) \nabla \Theta\right) d x=0$, we get

$$
\dot{E}=\int J . \nabla V d x-\int \nabla V . J d x=0 .
$$

4.2. The second law. For the second law, we note that the limit of the Shannon entropy is

$$
S=-\int f \log f d x-\int\left(f_{m}-f\right) \log \left(1-f / f_{m}\right) d x+\int f \log \Theta d x
$$

its time derivative is thus

$$
\begin{aligned}
\dot{S} & =-\int \dot{f} \log f d x-\int(f / f) \dot{f} d x-\int(-\dot{f}) \log \left(1-f / f_{m}\right) d x \\
& +\int \dot{f} / f_{m} d x+\int \dot{f} \log \Theta d x+\int f \dot{\Theta} / \Theta d x .
\end{aligned}
$$

Now, $\int \dot{f} d x=0,-\dot{f}=\operatorname{div} J$, and $\int \dot{f} \Theta / \Theta d x=0$. So we may replace $\int f \dot{\Theta} / \Theta d x$ by $\int(f \Theta) \cdot / \Theta d x$, for which we have eq. (3) or eq. (47). Then we get

$$
\frac{d S}{d t}=\int \operatorname{div} J\left(\log f-\log \left(1-f / f_{m}\right)\right) d x-\int \operatorname{div} J \log \Theta d x+\int(f \Theta) \cdot / \Theta d x .
$$

We impose boundary conditions so that surface terms vanish. Then the term

$$
-\int \operatorname{div} J \log \Theta d x=\int J . \nabla \Theta / \Theta d x
$$

cancels the term in $(f \Theta)$. of the form $-\int \Theta^{-1} \operatorname{div}(\Theta J) d x$. The rest can be rearranged to give

$$
\begin{aligned}
\frac{d S}{d t} & =\lambda^{-1} \int\left(f(x, t)\left(1-f(x, t) / f_{m}\right)\right)^{-1} J . J d x \\
& +\lambda \int f(x, t)\left(1-f(x, t) / f_{m}\right) \nabla \Theta . \nabla \Theta / \Theta^{2} d x \\
& \geq 0,
\end{aligned}
$$

since we assume that the solution obeys $0<f<f_{m}$. We see that such a solution is stationary only if $\nabla \Theta=0$ and $j=0$.

It can be seen that both laws of thermodynamics hold if $\lambda$ is a function of space and time, or depends on $\Theta$. In particular, the choice $\lambda=\kappa \Theta$ leads to a nonlinear analogue of Smoluchowski's equation.

5. A model of measurement. In measuring a quantum observable such as the position $q$ of a particle, we ideally measure instead the value of a classical variable, such as the position of a pointer, that is $100 \%$ correlated with $q$; we then infer the value of $q$ from our measurement. In practice we measure one or more observables $N_{1}, N_{2}, \ldots$, and in the simplest case, these are compatible with $q$, and all measured at the same time, $t$ say. The interpretation of the result is theory-dependent; we assume that the joint probability distribution $f\left(q, n_{1}, n_{2}, \ldots\right)$ of the observables is known or given by theory. Then the measurement of $N_{1}, N_{2}, \ldots$ allows us to replace the original distribution by the conditional distribution

$$
f(q \mid N)=f\left(q \mid N_{1}=n_{1}, N_{2}=n_{2}, \ldots\right) ;
$$


if $q$ is strongly correlated with the variables $N_{1}, N_{2}, \ldots$, then $f(q \mid N)$ is very strongly peaked about some value, so we almost know $q$ after the measurement has been made. On the other hand, if $Q$ is more or less independent of all the $N_{k}$, then we learn nothing about $q$ by the measurement. In a cloud chamber, a particle leaves a trail of ionized fluid behind, and this forms bubbles, which can be seen; in a spark chamber, a particle moves under an electric force, and leaves a wake of observable photons. The position of the particle is strongly correlated with these macroscopic traces. The measurement theory of such a device should therefore exhibit this correlation. For this, the models of a Brownian particle described in [14] are not useful, since the correlation between the particle and the heat is treated as a fast variable. This means that it is put to zero by the map $Q$, after each time-step. It could not be a good description of a spark chamber. The present model is therefore better in this respect. However, we would like to describe the measurement of a single particle, rather than a dense gas. In the present model, the state at time $t$ is described by a grand canonical state, so even if the mean density is taken to be that of one particle in the experimental volume, there is some uncertainty concerning the exact number of particles. This is not a bad description of the measurement of position for a beam of particles whose position along the beam is unknown.

Consider the present model with mean density equal to one particle in the experimental volume, so that we can ignore the term $\rho / \rho_{m}$. The equation for $\rho$ then becomes linear in $\rho$, and we might try to use it to describe the probability distribution of a beam. Suppose that in the initial state, the density is uniformly distributed along a finite interval of the the $x$-axis; because we use the grand canonical state, we cannot be sure of the exact number of particles present. Suppose that no measurement is made. According to the dynamics, the state will move to equilibrium with potential $V$ as time goes by; after a long time, the particles will on average be distributed according to the grand canonical state with constant temperature, and a density-field determined by $V$. We shall not know with certainty how many particles are present. If instead of making no measurement, a measurement of kinetic energy is made at a certain time $t$, and it is found to be nonzero at $x$, then the conditioned probability asserts that there is, with probability 1 , a particle at $x$. This is because every sample point with nonzero kinetic energy at $x$ has a particle at that point (but we cannot be sure that there are not other particles present elsewhere). Thus, the model is a possible instrument for measuring position. This contrasts with the model dynamics given in [14], and also with the classical model given in [11], page 76 . The latter is again a model with a hard core, but unlike [10] the particle is slowed by exchanging potential energy with the quanta of a boson field, and the heat is held by the field of oscillators, interpreted as excitations of the ambient fluid. An easy application of the methods in the present paper to the discrete equations [11], eq. (4.33) leads to the coupled equations

$$
\begin{aligned}
& \frac{\partial \rho}{\partial t}=\lambda\left\{\nabla^{2} \rho+\operatorname{div}\left(\frac{F(x)}{\Theta}\left(\rho\left(1-\rho / \rho_{\max }\right)\right)\right\},\right. \\
& \frac{\partial \Theta}{\partial t}=F .\left(-\nabla \rho+F \rho\left(1-\rho / \rho_{\max }\right) / \Theta\right) .
\end{aligned}
$$

This fails to have any convection, as there is no term in the heat flow proportional to the 
density of the fluid. It is easy to show that the dynamics given by these equations obeys both laws of thermodynamics. This model is useless for finding the position from a measurement of local temperature, (by measuring the photon number at $x$ ). This is because here the map $Q$ ensures that at each time, the position of the particle is statistically independent of the photon number at nearby bonds, even though the mean energy is conserved in time. Physically, this is explained by saying that the heating at the point $x$ is caused by all the particles in the gas, rather than by any one of them whose position we want to measure. No wonder, then, that measuring the photon number tells us nothing about the position of a chosen particle.

6. Acknowledgements. This work was started at the University of Madeira, May 1997. I thank Prof. Benilov for the hospitality of the Dept., and H. Nencka for arranging the visit. The work was completed at the Conference on Quantum Probability Gdańsk, July 1997; I thank R. Alicki and W. A. Majewski for the invitation, and the City of Gdańsk for hospitality.

\section{References}

[1] S.-i. Amari, Differential Geometric Methods in Statistics, Lecture Notes in Statistics, 28, Springer-Verlag, Berlin, 1985.

[2] R. Balian, Y. Alhassid and H. Reinhardt, Physics Reports 131, 2-146, North Holland (1986).

[3] A. Fick, Phil. Mag. 10 (4th series) (1855), 30-35.

[4] H. Hasagawa, Rep. Math. Phys. 33 (1993), 87.

[5] H. Hasagawa, Noncommutative extension of the information geometry, in: Quantum Communication and Measurement, Eds. V. P. Belavkin, O. Hirota, and R. L. Hudson, Plenum Press, New York, (1995); 327.

[6] H. Hasagawa, Rep. Math. Phys. 39 (1997), 49-68.

[7] H. Nagaoka, IEICE Tech. Report 89 (1989), 9.

[8] H. Nagaoka, Differential geometrical aspects of quantum state estimation and relative entropy, in: Quantum Communication and Measurement, Eds. V. P. Belavkin, O. Hirota and R. L. Hudson, Plenum Press, new York (1995), 449.

[9] R. F. Streater, Statistical Dynamics, Rep. Math. Phys. 33 (1993), 203-219.

[10] R. F. Streater, Convection in a gravitational field, J. Stat. Phys. 77 (1994), 441-448.

[11] R. F. Streater, Statistical Dynamics, pp 275, Imperial College Press, 1995.

[12] R. F. Streater, Information Geometry and Reduced Quantum Description, Rep. Math. Phys. 38 (1996), 419-436.

[13] R. F. Streater, Statistical Dynamics and Information Geometry, in: Geometry and Nature, Eds. H. Nencka and J.-P. Bourguignon, Contemporary Mathematics 203, 117-131, 1997. Amer. Math. Soc.

[14] R. F. Streater, A gas of Brownian particles in statistical dynamics, J. Stat. Phys. 88 (1997), 447-469.

[15] R. F. Streater, Nonlinear heat equations, to appear in Rep. Math. Phys.

[16] R. F. Streater, Dynamics of Brownian particles in a potential, J. Math. Phys. 38(9) (1997), 4570-4575. 